\title{
Development of an attention-touch control for manual cervical distraction: a pilot randomized clinical trial for patients with neck pain
}

\author{
M. Ram Gudavalli ${ }^{*}$, Stacie A. Salsbury ${ }^{1}$, Robert D. Vining ${ }^{1}$, Cynthia R. Long ${ }^{1}$, Lance Corber ${ }^{1}$, Avinash G. Patwardhan ${ }^{2}$
} and Christine M. Goertz ${ }^{1}$

\begin{abstract}
Background: Manual cervical distraction (MCD) is a traction-based therapy performed with a manual contact over the cervical region producing repeating cycles while patients lie prone. This study evaluated a traction force-based minimal intervention for use as an attention-touch control in clinical trials of MCD for patients with chronic neck pain.
\end{abstract}

Methods: We conducted a mixed-methods, pilot randomized clinical trial in adults with chronic neck pain. Participants were allocated to three traction force ranges of MCD: low force/minimal intervention (0-20 N), medium force (21-50 N), or high force (51-100 N). Clinicians delivered five treatments over two weeks consisting of three sets of five cycles of MCD at the C5 vertebra and occiput. Traction forces were measured at each treatment. Patient-reported outcomes included a pain visual analogue scale (VAS), Neck Disability Index (NDI), Credibility and Expectancy Questionnaire (CEQ), and adverse effects. A qualitative interview evaluated treatment group allocation perceptions.

Results: We randomized 48 participants, allocating an average of five each month. Forty-five participants completed the trial with three participants lost to follow-up. Most participants were women $(65 \%)$ and white $(92 \%)$ with a mean (SD) age of 46.8 (12.5) years. Mean traction force values were within the prescribed force ranges for each group at the C5 and occiput levels. Neck pain VAS demonstrated a benefit for high traction force MCD compared to the low force group [adjusted mean difference 15.6; 95 \% confidence interval (Cl) 1.6 to 29.7]. Participants in the medium traction force group demonstrated improvements in NDI compared to the low force group (adjusted mean difference 3.0; $95 \%$ $\mathrm{Cl} 0.1$ to 5.9), as did participants in the high traction force group (adjusted mean difference $2.7 ; 95 \% \mathrm{Cl}-0.1$ to 5.6). CEQ favored the high force group. Most low force participants correctly identified their treatment allocation in the qualitative interview. No serious adverse events were documented.

Conclusions: This pilot study demonstrated the feasibility of a clinical trial protocol and the utility of a traction-based, minimal intervention as an attention-touch control for future efficacy trials of MCD for patients with neck pain.

Trial registration: ClinicalTrials.gov NCT01765751 (Registration Date 30 May 2012)

Keywords: Adverse effects, Chiropractic, Clinical trial, Control groups, Forces, Traction, Manual therapy, Musculoskeletal manipulations, Neck pain, Outcome assessments, Traction

\footnotetext{
* Correspondence: gudavalli_r@palmer.edu

${ }^{1}$ Palmer Center for Chiropractic Research, Palmer College of Chiropractic, 741

Brady Street, 52803 Davenport, IA, USA

Full list of author information is available at the end of the article
}

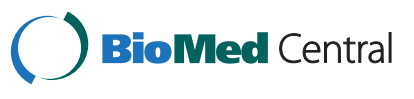

(c) 2015 Gudavalli et al. This is an Open Access article distributed under the terms of the Creative Commons Attribution License (http://creativecommons.org/licenses/by/4.0), which permits unrestricted use, distribution, and reproduction in any medium, provided the original work is properly credited. The Creative Commons Public Domain Dedication waiver (http:// creativecommons.org/publicdomain/zero/1.0/) applies to the data made available in this article, unless otherwise stated. 


\section{Background}

Chronic neck pain is a common musculoskeletal complaint associated with radiating symptoms to the head, upper extremities or thoracic regions, muscle stiffness, sensorimotor dysfunction, headache, vertigo, and psychological complaints [1-4]. The 12-month prevalence of neck pain in adults is estimated at $30 \%$ to $50 \%$ [5], with symptoms reported more frequently by women and middle-aged people [6-8]. Health service utilization among patients with neck pain is substantial, with 10.2 million visits to US healthcare facilities registered in 2007 [9].

Pharmacological and surgical interventions for chronic neck pain are available $[9,10]$, but many procedures are costly, leading to rising healthcare expenditures for this condition [11, 12]. In addition, non-steroidal anti-inflammatory medicines, opioids, spinal injections, and spine surgery have high-risk side effects [13-15] and may deliver only marginal improvements to patients' clinical outcomes, workrelated productivity and quality of life measures [11, 12]. Preliminary evidence suggests that manual therapies may provide similar benefits to patients with chronic neck pain as conventional medical treatments, with better tolerance and safety profiles [16-19]. However, a recent systematic review recommends additional studies to determine the efficacy of specific manual therapies to clinical outcomes in this patient population [20].

Clinical studies involving mechanical or manual therapies have demonstrated mixed clinical outcomes in patients with chronic neck pain [21-25]. These conflicting results are likely due to varying study methodologies and quality levels, preventing a conclusive determination of the efficacy of mechanical traction and other manual therapies for the relief of neck pain [26-31]. To improve the quality of such studies, recent systematic reviews have encouraged researchers to develop sham or minimal interventions to serve as control groups for clinical trials of manual treatments for neck pain [17, 32]. Toward this aim, our team conducted a series of basic science and clinical studies to develop a minimal intervention to serve as an attention-touch control group for efficacy trials of manual cervical distraction (MCD).

MCD is an intermittent traction procedure that differs from mechanically based traction therapies. MCD is delivered using a manual contact over different vertebral levels of the cervical spine in slow, repeating phases while the patient lies prone, whereas mechanical traction uses a head harness attached to weights or computer controlled tensioning equipment with patients in supine or upright positions [33]. MCD is hypothesized to create intersegmental separation at targeted vertebral levels, which contributes to generating a therapeutic effect [34]. Although MCD is used in the care of persons with neck pain [35-39], no published clinical trials have evaluated the efficacy of this therapy, in part due to the lack of adequate sham treatments or control intervention.

Our investigative team conducted a series of preliminary studies to develop a sham treatment or minimal intervention to serve as an attention-touch control for randomized controlled trials of MCD. Our objective in these studies was to identify cervical traction force ranges that demonstrated no intradiscal pressure decreases, but that patients with neck pain believed to be a credible treatment. In the first preliminary study, Gudavalli and colleagues (2013) confirmed that MCD decreased cervical spine intradiscal pressures in cadaveric specimens, even with traction forces as light as $20 \mathrm{~N}$ [40]. We then evaluated the perceived sensations of participants undergoing $\mathrm{MCD}$ to identify force ranges that participants classified as nontherapeutic, possibly therapeutic, and definitely therapeutic, with patients confirming $25.5 \mathrm{~N}$ as a possibly therapeutic level of traction force [41]. Based on the results of these preliminary studies, we concluded that developing a low-force minimal intervention will be a better choice than a sham treatment. We next developed a force feedback technology [42] to allow clinicians to deliver MCD within distinct traction force ranges, and tested a training and certification process using this technology [43]. These data led us to conclude that MCD could be delivered consistently within three different traction force ranges [44]. Our team then assessed clinician proficiency in delivering specified traction forces during a pilot randomized clinical trial (RCT) of MCD $[45,46]$.

The primary objective of this investigation was to evaluate a traction force-based, minimal intervention for patients with neck pain to use as an attention-touch control in future fully powered randomized trials studying MCD. This article also reports additional findings from this pilot RCT, including the feasibility of our participant recruitment plan and treatment protocol, the results of patient-reported outcomes (PROs), traction forces delivered, and patient perceptions of the three force-based treatment groups. Our presentation follows recent commentaries on reporting results of clinical trial pilot studies $[44,47]$.

\section{Methods/design}

This pilot RCT with a nested qualitative study compared three traction force ranges of MCD in 48 adults with chronic neck pain. Participants were randomized to one of three study groups: 1) low force/minimal intervention [0-20 Newtons $(\mathrm{N})], 2)$ medium force $(21 \mathrm{~N}-50 \mathrm{~N})$, or 3) high force $(51 \mathrm{~N}-100 \mathrm{~N}) \mathrm{MCD}$. At each treatment visit, participants received three sets of five repetitions of traction with a neutral head position, delivered within the allocated force range and at two contact points (C5 and occiput). Participants completed five study visits over two weeks, with PROs collected at baseline visit 1 (BL1) 
and before receiving treatment on the first and fifth study visits. Primary PROs included the Visual Analogue Scale (VAS) for neck pain and the Neck Disability Index (NDI). Secondary PROs included the Patient Reported Outcomes Measurement Information System (PROMIS43) general health status scale and patient satisfaction. Patient perceptions of study treatments and adverse events also were assessed. We evaluated participant blinding to their study intervention in a qualitative interview conducted after receiving their fifth MCD treatment on the last study visit.

\section{Ethical considerations}

The Palmer College of Chiropractic Institutional Review Board approved the study protocol and human subjects protection procedures for this trial (Approval \#2012G151, date 1 November 2013). Study coordinators provided an overview of the research procedures and played a videorecording that demonstrated the study procedures. The RCT was not identified as a pilot study in the consent; however, the written document identified all required elements for informed consent [48]. All participants read and signed the written consent document before the BL1 evaluation.

\section{Participant eligibility and recruitment}

We recruited participants of both genders, 18- to 70-years old using strategies that were successful in previous studies, including direct mail postcards, media releases, and internet postings [49]. Our targeted sample size was 45 participants. Participants completed a multi-stage eligibility determination process that included a telephone screen; in person informed consent process, questionnaires, clinical interview and examination by a Doctor of Chiropractic (DC); and a panel-based case review [50]. Eligible participants had self-reported neck pain or neck-related upper extremity pain of at least four weeks duration ranging in intensity from 3 to 7 on an 11-point (0 to 10) numerical rating scale (NRS) on the telephone screen [51]. Participants were not excluded for previous treatment of this episode of neck pain. However, because our primary aim was to develop a minimal intervention for MCD, participants could not have had any previous experience receiving flexion-distraction technique to the cervical spine. Table 1 displays each eligibility criterion with the corresponding rationale. Participant progress through the trial was recorded via a secure, password-protected, webbased tracking system according to CONSORT 2010 standards [52].

\section{Setting}

This study was conducted at the Palmer College of Chiropractic, Palmer Center for Chiropractic Research, in Davenport, IA, USA. The dedicated research clinic is a 250 square meter (2,700 square foot) facility with consultation, exam, and treatment rooms, clinical biomechanics laboratory and radiology suite. The clinic is staffed with research clinicians who are licensed DCs, study coordinators, and a research participant coordinator. The laboratory is staffed by biomechanics faculty and research assistants. Participants completed data collection and interviews in a private consultation room. Treatments and biomechanical measurements took place in a single room equipped with the treatment table and computerized biomechanical instrumentation, and staffed with up to three research personnel.

\section{Study visits}

Volunteers were screened by telephone and, if eligible, scheduled for a baseline visit (BL1) that included the informed consent process, baseline measures, an interview by a study coordinator, medication and health history review, an eligibility exam by a DC and, if indicated, a cervical radiographic evaluation. BL1 eligible participants were presented by the examining clinician to the case review panel, which rendered an eligibility determination [50]. The case review panel consisted of a co-investigator, project manager, clinicians, and study coordinators. Eligible participants at a second baseline visit (BL2) were assigned at random to one of three intervention groups and completed study visit 1 (SV1). Participants completed five study visits scheduled at least one day apart over two weeks with no more than three study visits during any single week. Study visits included questionnaires, clinical assessments, group-specific interventions, and specified research measurements.

\section{Treatment group allocation}

Participants were allocated to one of three treatment groups with a predetermined blocked randomization scheme with random block sizes of three or six created with computerized random numbers in a 1:1:1 ratio. A research clinician entered the participant identification number into the study-specific, web application that generated the coded treatment group, which was known only to the clinician. This web application algorithm concealed future group assignments.

\section{Intervention}

MCD is a form of low velocity variable amplitude spinal manipulation [34]. Figure 1 depicts the instrumented Cox Flexion-Distraction Table (Version 7, Haven Innovations Inc., Detroit, MI, USA) used for all trial treatments. The table includes a moveable headpiece that allows for guided head movement in multiple directions; however, only cephalic movement (traction) with the head in a neutral position was used in this study. During MCD, the participant laid prone on the treatment table while the clinician gently grasped the posterior aspect of the participant's 
neck with a broad contact (contact hand) between the thumb and index finger at a specific vertebral level (standardized at $\mathrm{C} 5$ and occiput). With the opposite hand, the clinician held a control handle attached to the headpiece. Using the contact hand, the clinician exhibited superior traction while ensuring gentle headpiece movement via the control handle. The weight of the participant's head and the light pressure from the manual contact was sufficient to maintain contact with the headpiece during the traction movement. The procedure produced a rhythmic, localized distractive movement lasting one to three seconds per repetition. Based on the findings of a preliminary study of patient perceptions of MCD traction forces [41], we limited cervical traction forces to less than or equal to

Table 1 Eligibility criteria

\begin{tabular}{ll}
\hline Inclusion criteria & Rationale \\
\hline $\begin{array}{ll}\text { Neck or neck-related upper limb pain consistent with Quebec Task } \\
\text { Force (QTF) classifications 2-4 [75] }\end{array}$ & $\begin{array}{l}\text { Study intervention was designed to treat radiating neck pain (proximal or dista } \\
\text { extremity) with or without neurological signs }\end{array}$ \\
Naïve to manual cervical distraction procedures to cervical & Treatment credibility assessment requires unfamiliarity with study interventions
\end{tabular}
spine region

\section{Exclusion criteria}

Neck pain without radiation (QTF classification 1) [75]

Presumptive compression of a nerve root on roentgenogram (i.e, instability fracture) (QTF classification 5)

Compression of spinal nerve root confirmed with imaging (QTF classification 6)

Spinal stenosis (QTF classification 7)

Postsurgical status $<6$ months (QTF classification 8)

Postsurgical status $>6$ months (QTF classification 9)

Chronic pain syndrome (QTF classification 10)

Other diagnoses from visceral disease, metastasis, etc. (QTF classification 11)

Inflammatory arthritis in the cervical spine: i.e., rheumatoid arthritis, systemic lupus erythematosus

Neurological (spinal) instability in cervico-thoracic spine

Tumors, within or adjacent to the cervico-thoracic spinal canal

Arnold Chiari malformation

Spinal joint hypermobility, such as: Marfan syndrome,

Ehlers-Danlos syndrome, osteogenesis imperfecta

Advancing neurologic deficits

Sequestered intervertebral disc or loose body within the cervical spinal canal, lateral recess, or intervertebral foramen

Fusion (single or multisegmental) of the cervical vertebrae

Safety precaution (e.g., unable to ambulate within clinic, dizziness, weight beyond treatment table limits [300 lbs])

Unable to tolerate study procedures

Simultaneous management for a condition compromising ability to deliver study treatment or assess health status

Suspicion of alcohol or drug abuse

Cognitive or memory impairment

Referral for evaluation/management of other condition(s) or neck pain diagnosis

Compliance concerns

\section{Rationale}

Intervention designed to treat radiating neck pain

May require individualized treatment not available in trial

May require individualized treatment not available in trial; condition may limit interpretation of study measurements

May require individualized treatment not available in trial; condition may limit interpretation of study measurements

May require individualized treatment not available in trial; condition may limit interpretation of study measurements

May require individualized treatment not available in trial; condition may limit interpretation of study measurements

Care needed is outside study scope

Care needed is outside study scope

Study treatments are not intended for these conditions

Treatment needed is outside study scope

Treatment needed is outside study scope

May require referral, additional evaluation or treatment outside study scope

May interfere with data collection and interpretation

Treatment needed is outside study scope

Safety precaution

Joint distraction and intervertebral disc pressure change are hypothesized as principal therapeutic mechanisms

Safety precaution

Safety precaution

Safety precaution and may present an undue scheduling burden

May interfere with data collection, ability to comply with study protocol, and requires referral

May prohibit informed consent or compromise safety due to potentially reduced comprehension or compliance with study procedures

Safety precaution and advanced diagnostic testing outside study scope

Transportation issues, scheduling conflicts, etc. may compromise adherence to study protocol 


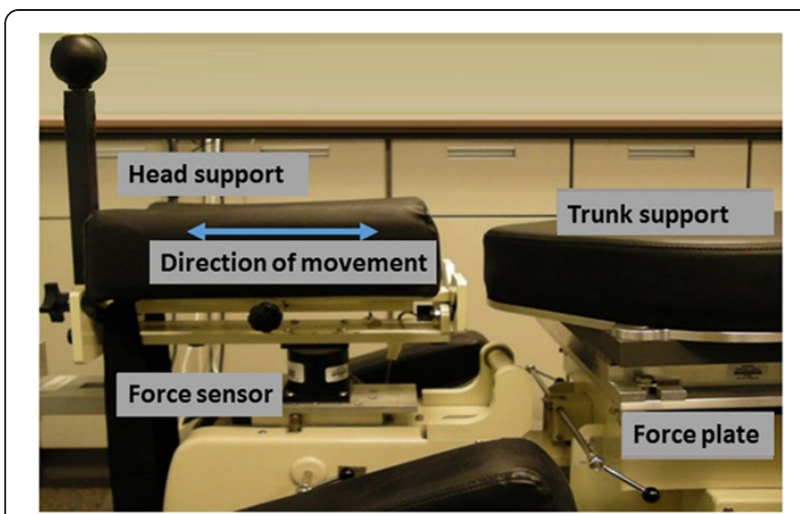

Fig. 1 Head and thorax support sections of instrumented manual distraction treatment table used in the study

$20 \mathrm{~N}$ in the low force group, between $21 \mathrm{~N}$ and $50 \mathrm{~N}$ in the medium force group, and between $51 \mathrm{~N}$ and $100 \mathrm{~N}$ in the high force group. Dosing was limited to three sets of five repetitions with contact over the $\mathrm{C} 5$ cervical vertebra and three sets of five repetitions with contact on the occiput. To insure inter- and intra-clinician proficiency in treatment implementation [46], we conducted pre-trial training sessions and monthly clinician recertification on traction force delivery using a process described elsewhere [43].

Participants wore a collarless shirt and placed their hands under their thighs instead of on the table hand rests, if able, to assure consistent positioning and force measures between participants and study visits (Fig. 2). All moveable sections of the table were locked in place except for the axial (cephalic) movement mechanism of the headpiece. We used only traction with a neutral head position (Cox protocol 1) [34] to standardize treatment delivery between participants and study visits.

\section{Measurements}

Additional measures were as follows. A verbal 0 to 10 numerical rating scale for pain was used at the telephone screen for eligibility determination due to its ease of administration for data collection [51]. We collected demographics at the phone screen and at the BL1. We assessed participants at BL1 for severe depression using the Beck Depression Inventory [53]. Study coordinators completed a medication checklist with participants at SV1 and SV5. PROs and questionnaires to assess treatment credibility and participant expectations were collected on participant-administered paper forms at BL1 and before treatment delivery at SV1 and SV5. Cervical traction forces were collected during each treatment. Cervical range of motion was measured at SV1 and SV5. A qualitative interview was completed after other study procedures on SV5. There were no follow-up assessments.

\section{Patient-reported outcomes}

PROs included the Visual Analogue Scale (VAS), Neck Disability Index (NDI), the Patient Reported Outcomes Measurement Information System (PROMIS-43) general health status scale, and patient satisfaction. The VAS is a single-item, unidimensional measure for pain with excellent metric properties, easy administration and scoring that is commonly used in pain-related research [51]. Our anchors for current neck pain were 'no pain' to 'worst imaginable pain' using a 0 to $100 \mathrm{~mm}$ scale, which allowed for more sensitive assessment of pain intensity compared to the NRS due to its increased number of response levels [54]. Score interpretations for the VAS include the following cut points: no pain ( 0 to $4 \mathrm{~mm}$ ), mild pain (5 to $44 \mathrm{~mm}$ ), moderate pain ( 45 to $74 \mathrm{~mm}$ ), and severe pain (75 to $100 \mathrm{~mm}$ ) [55]. The NDI is a 10-item questionnaire modified from the Oswestry Disability Index for low back pain $[56,57]$. The NDI is a reliable and valid measure of disability due to neck pain, and responsive for measuring change on a scale of 0 to 50 . Following the Initiative on Methods, Measurement and Pain Assessment in Clinical Trials (IMMPACT) recommendations for chronic pain clinical trials, we measured several additional core outcomes, including physical and emotional function [58] using the PROMIS-43 to evaluate pain interference,

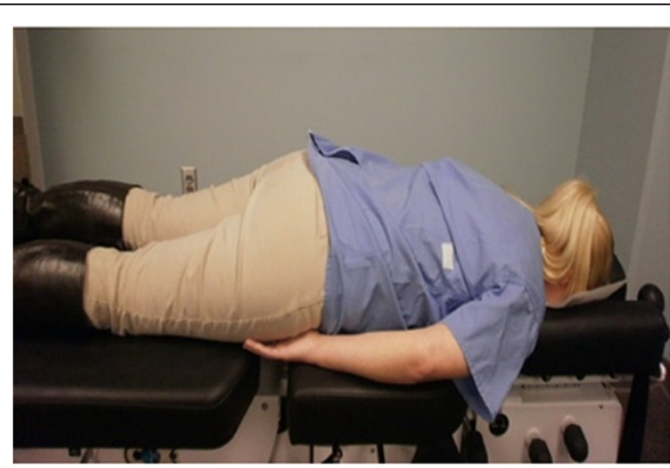

a. Patient in prone position

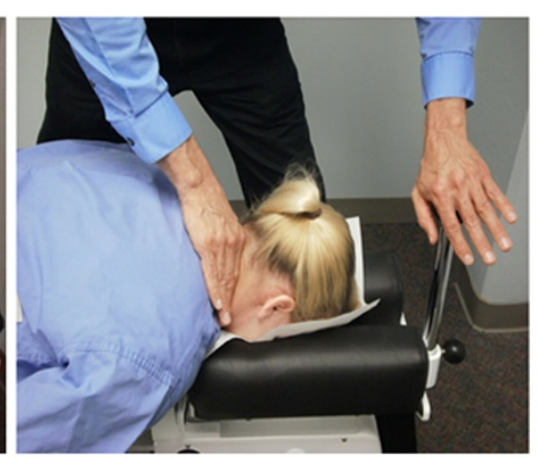

b. $\mathrm{C} 5$ contact

Fig. 2 Photographs showing (a) participant lying prone on treatment table and (b) clinician hand contact at C5 vertebral level 
physical function, fatigue, and sleep disturbance [59]. Participants rated their satisfaction with various elements of the study (e.g., treatments received, clinicians) using a 5 category response from 1 (strongly satisfied) to 5 (strongly dissatisfied) with categories collapsed into satisfied (categories 1 and 2) or not satisfied (categories 3, 4 or 5) for interpretation.

\section{Cervical traction forces}

A research assistant trained in biomechanical measurements recorded cervical traction forces during all study visits. The treatment table was modified to include force plates and motion sensors (Model 4060-1000, Bertec Inc., Columbus, OH, USA) to measure traction forces and table movement. Force plates were connected to a computer through an analog-to-digital converter. Motion Monitor Software (Version 7.1, Innovative Sports Training Inc., Chicago, IL, USA) collected data from the force plates and motion sensors at a sampling rate of $100 \mathrm{~Hz}$. Data were exported to text file using the export function of the software. Custom written Matlab software (Version 7, Mathworks Inc., Natick, MA, USA) read the exported data and graphed the forces as a function of the duration of treatment. A research assistant then used starting and end time points on the force-time graph to extract the peak traction forces between these time points. Peak forces data were exported from Matlab to an MS-Excel 2010 file (Microsoft Corporation, Redmond, WA, USA) and the average peak force in $\mathrm{N}$ was computed.

\section{Credibility and Expectancy Questionnaire}

Participants' perceptions of the study treatments were compared across groups using the Credibility and Expectancy Questionnaire (CEQ) [60, 61]. The CEQ credibility factor relates to how logical or believable a treatment is for the patient, whereas the expectancy factor refers to the patient's beliefs about improvements that might be achieved from the treatment [61]. CEQ scores for both the credibility and expectancy factors range from 3 to 27 , with higher scores indicating greater belief in treatment credibility or expectancy for clinical improvement. Psychometric evaluations of the CEQ in varying populations demonstrated high internal consistency (standardized $\alpha=$ 0.79 to 0.90 expectancy; Cronbach's $\alpha=0.81$ to 0.86 credibility; standardized $\alpha=0.84$ to 0.85 whole scale), good inter-item correlations (expectancy ranging from 0.53 to 0.85 ; credibility ranging from 0.62 to 0.78 ) and high testretest reliability over one-week (0.82 expectancy; 0.75 credibility) [61]. Participants completed the CEQ at the BL1 after reading the consent document and viewing the introductory video about $\mathrm{MCD}$, at the SV1 after receiving the MCD treatment for the first time, and at the SV5 before receiving their final treatment.

\section{Qualitative interviews}

As this was a pilot study evaluating a novel minimal intervention for use as an attention-touch control in future clinical trials, we assessed participants' perceptions of study treatments through standardized qualitative interviews, as recommended in a previous placebo-controlled trial of manual therapies [62]. One investigator (SAS) who has expertise in qualitative methods conducted all interviews with participants after their completion of the PROs, biomechanical measures, and MCD treatment at SV5. A professional transcriptionist transcribed the interviews verbatim. The interviewer was blinded to treatment group until after a question on treatment group assignment was asked during the interview schedule. Qualitative data were imported into NVIVO software (Version 9, QSR International Pty Ltd., Doncaster, Victoria, Australia) to facilitate data management and qualitative content analysis [63]. We appraised whether participants correctly identified their treatment group assignment (low force, medium force or high force) and their reasons for attributing treatment allocation to this group. Responses were broken down into discrete statements, marked with codes, and grouped into themes based on recurring patterns [64].

\section{Data management}

We have described our data management procedures elsewhere $[65,66]$. All data collection forms had unique participant identification numbers. Data forms were stored in locked filing cabinets or on a secure, password-protected server accessible only by study personnel. Data were double-key entered into computer databases that contain range and logic checks for accuracy. Electronic data were stored on an internal Microsoft SQL Server secured with Secure Socket Layers (SSL). Biomechanical data were stored on a secured network drive. Analytic and qualitative files were maintained on a secure, password-protected server.

\section{Sample size}

This pilot RCT was not a powered study. We targeted a sample size of 15 per group $(n=45)$ to provide adequate participant contact to evaluate study protocol feasibility and recruitment strategies and to assess the believability characteristics of the three traction force-based treatments. We also estimated group differences in mean changes and standard deviations in the outcome measures. These aspects of the study protocol were selected as key to informing the design of a full-scale efficacy trial of MCD.

\section{Statistical methods}

Participants who had non-missing data for the primary outcomes were analyzed according to their original treatment assignment. We performed quantitative data 
analyses using SAS/STAT version 9.3 (SAS Institute, Inc., Cary, NC, USA). Change in PROs from BL1 to SV5 (before biomechanical measurements and treatment) were compared between groups with analysis of covariance (ANCOVA), adjusting for the BL1 value of the respective variable centered at its mean. We then entered baseline variables that were imbalanced among groups, as well as a variable differentiating if the intervention was aided by visual feedback, into the models one at a time. Only variables significant at the 0.10 level were kept in the final models. Adjusted within group changes and between group differences in mean change and $95 \%$ confidence intervals based on the final ANCOVA models are presented.

\section{Blinding protocol}

Clinicians were not masked to group assignment, but were blinded to PROs. Study coordinators, examining clinicians, investigators and analysts were blinded to group assignment. Biomechanical technicians were blinded to group assignment but not traction forces, while research assistants completing data reduction were blinded to group assignment. The qualitative interviewer was blinded to group assignment during the exit interview until the participants were asked a specific question on treatment group assignment, at which point the participant and researcher were unmasked to treatment group. However, the qualitative researcher was masked to treatment group during data coding. Participants were masked to group assignment during the trial until questions on the exit interview specifically asked them to guess their treatment group assignment.

\section{Safety, protocol deviation and compliance monitoring}

Recent articles have called for more consistent, in-depth and standardized monitoring of adverse events related to manual therapies [67-71]. Toward that aim, we carefully monitored adverse events (AE) and serious adverse events (SAE) using an active surveillance process described elsewhere $[65,66]$. AEs were defined as any untoward medical occurrence that may present itself during the conduct of the study which may or may not have a causal relationship with the study procedures [72]. SAEs were defined as any adverse experience occurring during treatment that results in any of the following outcomes: death, life-threatening adverse experience, hospitalization, persistent or significant disability or incapacity, or a congenital anomaly or birth defect [72]. Clinicians collected AE data by directly querying participants about any new injuries, side effects or symptoms at each visit. Positive responses initiated the completion of an Adverse Event Report, with all reported AEs and SAEs graded on three criteria: 1) severity; 2) expectedness; and 3) relatedness to the study interventions. The informed consent document disclosed muscle soreness or stiffness, light-headedness or dizziness, headache and exacerbation of neck pain symptoms as AEs experienced by patients receiving manual therapy for neck pain [73, 74].

Protocol deviations were tracked using a study-specific, web application that included the date of occurrence, participant(s) involved, detailed event notes, and investigator response. Participant compliance was tracked through the scheduling database that included details on missed appointments and study dropouts.

\section{Results}

\section{Participant flow and recruitment feasibility}

Figure 3 presents the participant flowchart. We completed 291 telephone screens, with 210 participants eligible for the BL1 clinic screen. Ninety-six participants were presented at case review, with 60 participants eligible for the BL2 clinic screen. In total, 109 participants chose not to participate in the trial and 134 participants were excluded. The most common reasons participants did not enroll in the study were non-attendance at a scheduled appointment $(n=43)$, time or other outside commitments $(n=30)$, not interested $(n=16)$, not willing to stop treatment from another provider $(n=5)$, other health concern $(n=4)$, neck condition not requiring treatment at this time $(n=3)$, not willing to receive study treatments $(n=2)$, or non-specified reasons $(n=6)$. The most common exclusions were for a neck pain rating of $\leq 2$ on the NRS ( $\mathrm{n}=37$ ), a neck pain rating of $\geq 8$ on the NRS $(\mathrm{n}=32)$, current litigation $(\mathrm{n}=10)$, inability to tolerate study procedures during BL1 screening $(n=9)$, the need for referral or concurrent treatment identified during BL1 screening $(n=6)$, Quebec Task Force classification of neck symptoms outside the ratings of 2,3 , or $4(n=6)$, multiple exclusions $(n=17)$, or other exclusions $(17)$. We randomized 48 participants to treatment, allocating an average of five participants each month (range 1 to 10 participants/ month). Forty-five participants completed the trial and three participants were lost to follow-up (two medium force participants and one high force participant). We were unable to ascertain the reasons these three participants dropped out of the study. Only four participants missed any of the five scheduled treatment visits; of these, three participants were those who later withdrew from the study, missing two, three, and four treatments, respectively. We opted not to perform study treatments or biomechanical tests on one participant who experienced an adverse event (described below) following the first study visit. This participant did not attend two study visits, but completed all outcome measures.

\section{Baseline demographic and clinical characteristics}

Table 2 depicts the BL1 characteristics of participants by treatment group. Overall, most participants were women 


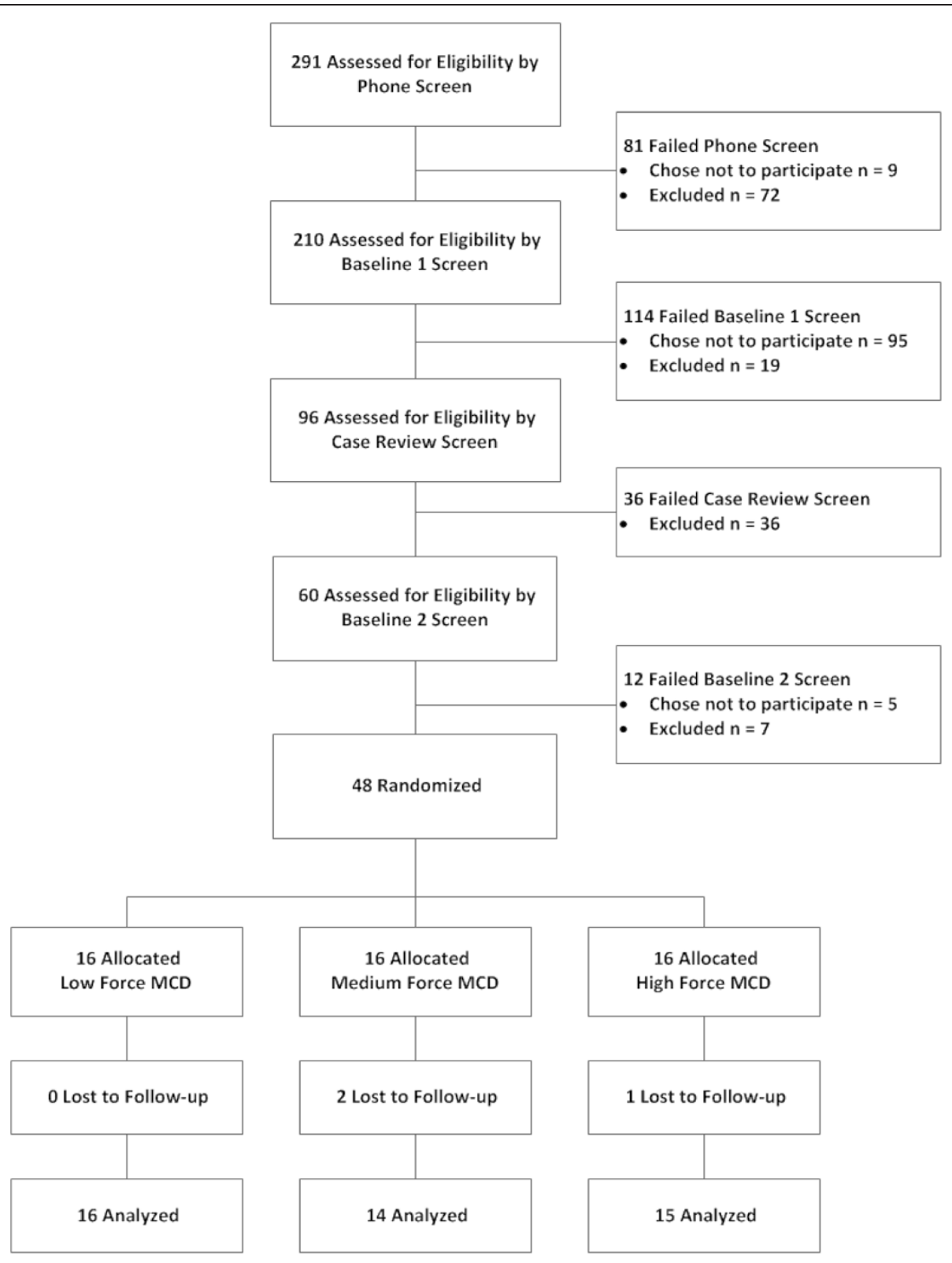

Fig. 3 Participant flow diagram

(65\%) and white (92\%) with a mean (SD) age of 46.8 (12.5) years. All participants were classified with neck pain consistent with category 2 (radiation to the proximal extremities) or 3 (radiation to distal extremities) of the Quebec Task Force for Spinal Disorders [75]. These categories are roughly equivalent to severity grades 1 and 2 proposed by the Neck Pain Task Force for patients with neck pain who seek healthcare [76]. Most participants (90\%) had received chiropractic care in the past, although only $25 \%$ of the sample considered themselves as someone who regularly receives chiropractic care.
Current neck pain on the VAS at BL1 for the sample was a mean (SD) of 41.3 (15.4) indicating mild neck pain [55]. Participants allocated to the higher force group reported lower levels of current pain at BL1 than participants in the other groups. The NDI scores for the sample measured a mean (SD) of 11.0 (4.1), consistent with a mild disability from neck symptoms [77]. Participants reported a mean duration of neck pain of 14 years, ranging from 1-45 years. Participants' self-reported cause of neck pain included a gradual onset $(n=17)$, no known cause $(n=8)$, office or desk work $(n=7)$, motor vehicle accidents $(n=6)$, 
Table 2 Participant characteristics at baseline

\begin{tabular}{|c|c|c|c|c|c|c|}
\hline \multirow{2}{*}{$\begin{array}{l}\text { Characteristic } \\
\text { Age in years, mean (SD) }\end{array}$} & \multicolumn{2}{|c|}{$\begin{array}{l}\text { Low Force } \\
(n=16)\end{array}$} & \multicolumn{2}{|c|}{$\begin{array}{l}\text { Medium Force } \\
(n=16)\end{array}$} & \multicolumn{2}{|c|}{$\begin{array}{l}\text { High Force } \\
(n=16)\end{array}$} \\
\hline & 42.2 & 11.2 & 47.0 & 11.4 & 51.2 & 13.7 \\
\hline Female, n (\%) & 11 & 69 & 12 & 75 & 8 & 50 \\
\hline White, n (\%) & 15 & 94 & 14 & 88 & 15 & 94 \\
\hline Married, n (\%) & 7 & 44 & 10 & 63 & 9 & 56 \\
\hline Education, high school graduate or higher, n (\%) & 16 & 100 & 16 & 100 & 15 & 94 \\
\hline Employment, full-time, n (\%) & 11 & 69 & 9 & 56 & 8 & 50 \\
\hline Previous chiropractic, n (\%) & 16 & 100 & 14 & 88 & 13 & 81 \\
\hline Regular chiropractic care, $\mathrm{n}(\%)$ & 3 & 19 & 5 & 31 & 4 & 25 \\
\hline Body mass index, mean (SD) & 31.3 & 6.9 & 30.3 & 6.3 & 26.9 & 5.3 \\
\hline Beck depression index, mean (SD) & 5 & 3.8 & 5 & 5.0 & 4 & 3.7 \\
\hline Current neck pain, Visual Analog Scale (0-100 mm), mean (SD) & 45.2 & 18.0 & 44.3 & 14.1 & 34.5 & 12.3 \\
\hline Neck disability index (0-50), mean (SD) & 10.8 & 4.0 & 11.1 & 4.5 & 11.1 & 3.9 \\
\hline \multicolumn{7}{|c|}{ Neck pain or symptom radiation pattern (may indicate more than 1 location of symptom radiation) } \\
\hline Head, n (\%) & 1 & 6 & 2 & 13 & 2 & 13 \\
\hline Thoracic, n (\%) & 3 & 19 & 2 & 13 & 4 & 25 \\
\hline Shoulder, n (\%) & 8 & 50 & 3 & 19 & 8 & 50 \\
\hline Upper arm, n (\%) & 1 & 6 & 0 & 0 & 2 & 13 \\
\hline Distal arm/hand, n (\%) & 3 & 19 & 8 & 50 & 2 & 13 \\
\hline \multicolumn{7}{|l|}{ Quebec Task Force Classification } \\
\hline QTF 2, n (\%) & 11 & 23 & 11 & 23 & 14 & 29 \\
\hline QTF 3, n (\%) & 5 & 10 & 5 & 10 & 2 & 4 \\
\hline PROMIS-pain interference, mean (SD) & 53.2 & 6.7 & 54.9 & 5.2 & 51.2 & 5.6 \\
\hline PROMIS-physical function score, mean (SD) & 27.7 & 5.2 & 25.3 & 5.0 & 26.4 & 4.5 \\
\hline PROMIS-sleep disturbance, mean (SD) & 51.3 & 3.0 & 51.3 & 3.0 & 51.5 & 2.6 \\
\hline PROMIS-fatigue, mean (SD) & 50.4 & 10.9 & 46.4 & 9.3 & 47.1 & 7.2 \\
\hline
\end{tabular}

PROMIS Patient-Reported Outcomes Measurement Information System, QTF 2 Quebec Task Force Rating 2 - pain and radiation to proximal extremity, QTF 3 Quebec Task Force Rating 3 - pain and radiation to distal extremity

sports injury $(\mathrm{n}=5)$, manual labor $(\mathrm{n}=3)$ or an accidental fall $(n=2)$. Participants reported a mean of 2.5 (range 0 to 7) neck pain aggravators, with frequent aggravators noted as seated posture $(n=18)$, reading $(n=15)$, computer use $(n=13)$, sleep position or quality $(n=9)$, driving $(\mathrm{n}=8)$, neck or head motions $(\mathrm{n}=8)$, lifting $(\mathrm{n}=8)$, stress $(n=8)$, motor vehicle accidents $(n=6)$, sports participation or injuries $(n=6)$, housework $(n=5)$, office or desk work $(\mathrm{n}=4)$, standing posture $(\mathrm{n}=2)$, weather change $(\mathrm{n}=2)$, music playing $(\mathrm{n}=2)$, and other aggravators $(\mathrm{n}=8)$.

\section{Patient-reported outcomes}

Pain intensity at baseline was significant $(\mathrm{p}=0.07)$ in the model for change in pain interference and, therefore, remained in the final reported model. The traction-force feedback method was significant in the models for change in VAS $(\mathrm{p}=0.06)$ and change in NDI $(\mathrm{p}=0.01)$, and remained in the final reported models. Sex and BMI were not significant in any of the models.

\section{Neck pain intensity}

The raw mean VAS at baseline and SV5 for each group is given in Fig. 4. Adjusted mean neck pain VAS change (95\% confidence interval) was $9.1 \mathrm{~mm}$ (-1.6 to $19.8 \mathrm{~mm}$ ), $18.9 \mathrm{~mm}$ (7.7 to $30.1 \mathrm{~mm}$ ), and $24.7 \mathrm{~mm}$ (13.9 to $35.5 \mathrm{~mm}$ ) for the low, medium and high force groups, respectively. The adjusted mean neck pain intensity change for the high traction force MCD group was significantly greater $(15.6 \mathrm{~mm})$ than the low force group (Table 3). The adjusted mean neck pain intensity change was $9.8 \mathrm{~mm}$ greater for those in the medium traction force MCD than those in the low force group, but there was little difference compared to the high force group $(5.8 \mathrm{~mm})$.

\section{Neck-related disability}

The raw mean NDI at baseline and SV5 for each group is given in Fig. 5. Adjusted mean NDI change (95 \% confidence interval) was 1.3 (-1.0 to 3.5 ), 4.2 (1.8 to 6.6), 


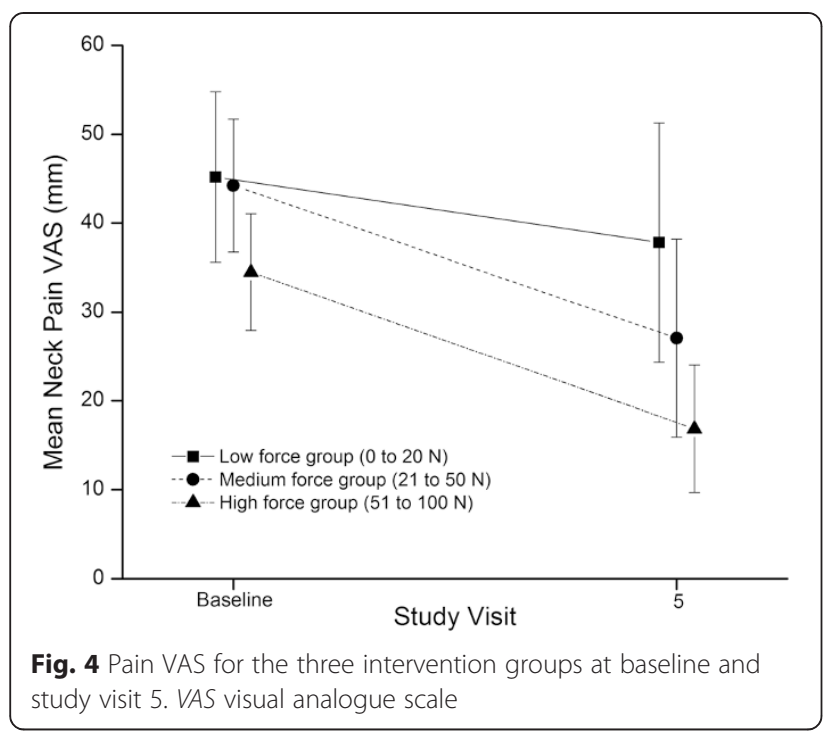

Fig. 4 Pain VAS for the three intervention
study visit 5. VAS visual analogue scale and 4.0 (1.8 to 6.2) for the low, medium and high force groups, respectively. The adjusted mean NDI change was approximately the same for the medium and high force groups, and both had significantly more change (2.7-3.0 points) than the low force group (Table 3).

\section{PROMIS-43 outcomes}

Adjusted mean differences on the PROMIS-pain interference and physical function were not appreciably different between groups (Table 3). However, the mean PROMIS fatigue change score was significantly greater for the high traction force MCD than the low force group (5.8).

\section{Satisfaction}

More high traction force participants $(\mathrm{n}=15)$ were satisfied with the treatments they received compared to participants in the medium $(n=9)$ or low traction force $(n=7)$ groups. In contrast, participants in all three groups were satisfied with the clinician who provided their care (high force group, $n=15$; medium force group, $n=14$; low force group, $\mathrm{n}=14$ ).

Table 3 Adjusted mean differences of patient-reported outcomes

\begin{tabular}{|c|c|c|c|}
\hline Outcome variable & Treatment group comparison & Mean difference ${ }^{a}$ & $95 \%$ Confidence interval $^{a}$ \\
\hline \multirow[t]{3}{*}{ Neck Pain VAS $(\mathrm{mm})^{\mathrm{b}}$} & High vs. Low & 15.6 & 1.6 to 29.7 \\
\hline & Medium vs. Low & 9.8 & -3.7 to 23.3 \\
\hline & High vs. Medium & 5.8 & -8.6 to 20.3 \\
\hline \multirow[t]{3}{*}{ Neck Disability Index } & High vs. Low & 2.7 & -0.1 to 5.6 \\
\hline & Medium vs. Low & 3.0 & 0.1 to 5.9 \\
\hline & High vs. Medium & 0.2 & -2.7 to 3.2 \\
\hline \multicolumn{4}{|l|}{ PROMIS - } \\
\hline \multirow[t]{3}{*}{ Pain Interference ${ }^{c}$} & High vs. Low & 3.9 & -5.6 to 2.7 \\
\hline & Medium vs. Low & -1.5 & 1.0 to 0.1 \\
\hline & High vs. Medium & 5.4 & -2.2 to 9.8 \\
\hline \multicolumn{4}{|l|}{ PROMIS - } \\
\hline \multirow[t]{3}{*}{ Physical Function } & High vs. Low & -0.2 & -2.2 to 1.9 \\
\hline & Medium vs. Low & -1.0 & -3.1 to 1.2 \\
\hline & High vs. Medium & 0.3 & -1.9 to 2.5 \\
\hline \multirow[t]{3}{*}{ PROMIS - Fatigue } & High vs. Low & 5.8 & 1.1 to 10.5 \\
\hline & Medium vs. Low & 3.3 & -1.5 to 8.2 \\
\hline & High vs. Medium & 2.5 & -2.3 to 7.3 \\
\hline \multicolumn{4}{|l|}{ PROMIS - } \\
\hline \multirow[t]{3}{*}{ Sleep Disturbance } & High vs. Low & 1.3 & -0.9 to 3.5 \\
\hline & Medium vs. Low & -1.4 & -3.6 to 0.9 \\
\hline & High vs. Medium & 2.7 & 0.4 to 5.0 \\
\hline
\end{tabular}

High High force group (51 to 100 Newtons), Low Low force group (0 to 20 Newtons), Medium Medium force group (21 to 50 Newtons), PROMIS Patient-Reported Outcomes Measurement Information System, VAS Visual Analog Scale

${ }^{a}$ Adjusted for the baseline value of the respective variable centered at its mean;

${ }^{\mathrm{b}}$ Also adjusted for traction-force feedback method; ' $\mathrm{C}$ Also adjusted for baseline neck pain VAS 


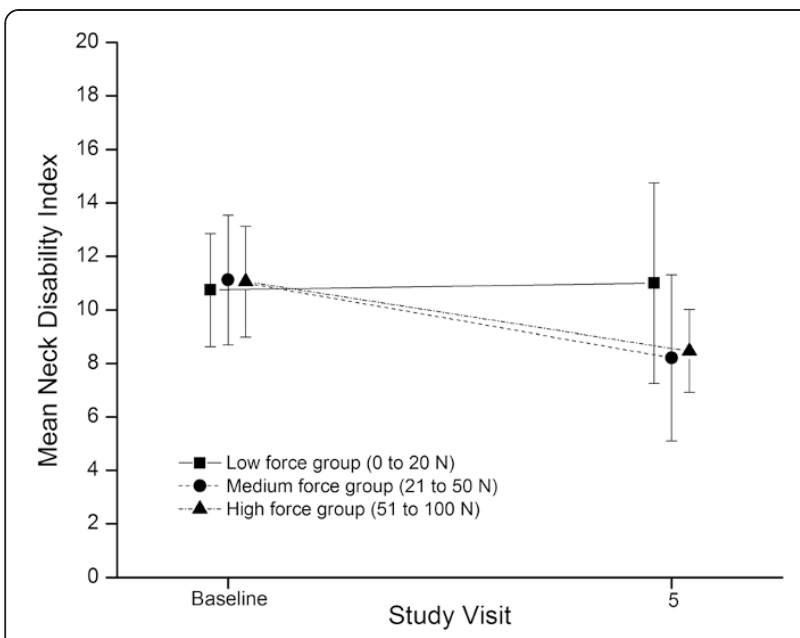

Fig. $5 \mathrm{NDI}$ for the three intervention groups at baseline and study visit 5. ND/ Neck Disability Index

\section{Cervical traction forces}

Table 4 presents the cervical traction forces delivered to participants. The mean values are within the prescribed force ranges for each group at both the C5 and occiput levels. Maximum forces were highest at the occiput level for all three force groups. Maximum values for the light force group reached $27 \mathrm{~N}$. Minimum values for the medium force group were as low as $8 \mathrm{~N}$ and as high as $70 \mathrm{~N}$ for the maximum values. Minimum force values for the high force group were $31 \mathrm{~N}$ and maximum force was $99 \mathrm{~N}$. In some cases, clinicians delivered MCD cycles outside the allocated force range.

\section{Treatment credibility and expectancy measures}

Table 5 depicts the changes in CEQ scores between treatment groups over the two-week trial. Credibility factor scores at BL1 suggest participants considered MCD, as described in the consent document and introductory video, to be a credible therapy for neck pain. After receiving MCD for the first time (SV1), participants in the high force group reported higher credibility scores from BL1, while credibility scores fell for medium and low force group participants. Credibility score trends continued at the final visit, where the high force group reported the highest credibility scores, the medium force group remained stable, and the low force group dropped six points from BL1. Expectancy factor scores followed similar patterns. Participants in the high force group reported greater expectancy scores after receiving MCD at SV1 compared to the other groups. However, expectancy scorings dropped for all treatment groups at SV5.

\section{Qualitative analysis of treatment group allocation}

Thirty-nine $(81 \%)$ participants completed a qualitative interview which included two questions that asked participants to identify their treatment group assignment. No interviews were conducted with the three participants who withdrew from the study (two medium force, one high force) while scheduling conflicts prevented the completion of six additional interviews (three low force, two medium force, one high force). We completed interviews with the majority of participants in each treatment group. With this number of interviews, we achieved consistent thematic coding of participants' attributions for treatment allocation within and across groups (largely related to the amount of pressure or force used by clinician during treatment delivery), suggesting we reached theoretical saturation on this topic [78].

In the low force group, 12 of 13 (92\%) participants correctly identified their treatment assignment as "low force'. Most participants noted the light touch used by the clinician as the reason for their treatment group attribution, as indicated by this participant: "It didn't feel like there was much pressure being put on there." One low force participant incorrectly identified the treatment allocation stating, "I believe I received the medium, but I'm not sure. It wasn't really tight, but it wasn't really soft either. It was sort of like an in-between." In the medium force group, 4 of 13 (31\%) participants correctly identified their treatment assignment as 'medium force,' with 7 participants incorrectly guessing their group (6 as low force, 1 as high force), and 2 participants identifying their group as 'low-to-medium' force. In the high force group, 2 of 13 (15\%) participants correctly identified their group allocation as high force, while 9 (69\%) participants incorrectly guessed their group (8 as medium force, 1 as low force), and 2 participants assessed

Table 4 Cervical traction forces measured in Newtons (N) over four treatment visits

\begin{tabular}{lllllll}
\hline Force range & Contact level & Number of observations ${ }^{\text {a }}$ & Mean & SD & Minimum & Maximum \\
\hline Light Force $(0-20 \mathrm{~N})$ & C5 & 64 & 13.82 & 5.74 & 0.18 & 23.31 \\
& Occiput & 64 & 17.19 & 4.84 & 0.47 & 26.64 \\
Medium Force $(21-50 \mathrm{~N})$ & C5 & 57 & 38.30 & 12.12 & 8.61 & 59.07 \\
& Occiput & 56 & 42.87 & 10.40 & 7.70 & 69.57 \\
High Force $(51-100 \mathrm{~N})$ & C5 & 60 & 65.08 & 17.70 & 30.63 & 89.65 \\
& Occiput & 60 & 74.06 & 16.08 & 37.54 & 98.34 \\
\hline
\end{tabular}

${ }^{a}$ We recorded 23 missing values for traction forces due to 9 missed appointments (18 observations) and 5 instances of technical problems in data collection 
that they were in a 'medium-to-high' force treatment group. The participants who correctly identified their treatment group as high force noted the clinicians' behavior as an indicator of their assignment, such as, "He (the chiropractor) seemed to exert himself a little bit". In contrast, high force participants who incorrectly guessed their group assignment expected more forceful sensations from the treatment, as did this participant: "He (the chiropractor) could have pushed harder, you know?"

\section{Participant safety}

No serious adverse events were documented. Fifty-seven adverse events were reported by 29 participants (including 1 non-randomized). However, 34 of the AEs reported by 23 participants were graded as unrelated $(n=30)$ or unlikely $(n=4)$ related to study interventions and were classified as mild $(n=32)$ or moderate $(n=2)$ in severity. Another 23 AEs were reported by 14 participants and graded as possibly $(n=1)$, probably $(n=6)$ or definitely $(n=15)$ related to study interventions. These related AEs were all classified as mild severity (that is, transient and minimal symptoms needing no change in activity level or additional treatment) and were characterized as neck or back soreness or pain $(n=14)$, muscle or joint stiffness $(n=3)$, combined pain and stiffness $(n=2)$, headache $(n=1)$, vertigo $(n=1)$, and pain paired with a flushed sensation $(n=1)$. One episode of vertigo that occurred within 30 hours of SV1 in a participant with previous episodes of dizziness was classified as a severe $\mathrm{AE}$ that was probably related to the study interventions. Of the 23 AEs attributed to the study, 3 AEs were reported by 3 participants assigned to the low force group, 15 AEs were reported by 6 participants assigned to the medium force group, and $5 \mathrm{AEs}$ were reported by 5 participants assigned to the high force group. Twenty randomized participants reported no adverse events, including nine low force, five medium force, and six high force participants.

Table 5 Credibility and expectancy questionnaire results

\begin{tabular}{lcccccc}
\hline & \multicolumn{2}{c}{ Baseline 1 } & \multicolumn{2}{c}{ Study visit 1 } & \multicolumn{2}{c}{ Study visit 5 } \\
& Mean & SD & Mean & SD & Mean & SD \\
\hline Credibility factor & & & & & & \\
Low force & 19.8 & 2.2 & 15.5 & 7.0 & 13.8 & 8.0 \\
Medium force & 23.1 & 3.4 & 19.9 & 5.2 & 19.9 & 7.5 \\
High force & 21.0 & 3.9 & 22.1 & 4.8 & 22.7 & 4.0 \\
Expectancy factor & & & & & & \\
Low force & 15.8 & 4.0 & 13.4 & 6.2 & 11.4 & 7.8 \\
Medium force & 19.7 & 4.7 & 17.8 & 5.8 & 15.1 & 8.3 \\
High force & 16.0 & 6.9 & 19.3 & 6.7 & 17.8 & 6.2 \\
\hline
\end{tabular}

\section{Protocol deviation}

We recorded one protocol deviation in this trial. Our original plan was to train research clinicians to deliver MCD at the three traction force ranges using tactile sensations alone, as manual therapists deliver treatments in clinical practice. During a monthly re-certification, investigators noted modest drift in the clinicians' delivery of MCD. Delivery of the low force group drifted into the medium range, particularly for forces delivered to the occiput, although traction forces remained under $25 \mathrm{~N}$. Medium traction forces drifted in each direction and at both contact levels. High traction forces drifted toward the medium range, for both vertebral levels, with most drift measured between $30 \mathrm{~N}$ to $50 \mathrm{~N}$; no traction forces were noted above $100 \mathrm{~N}$. To address this drift, the researchers developed a visual feedback tool to augment the auditory and graphical feedback received by clinicians during training (Motion Monitor software, Version 7.1, Innovative Sports Training, Inc.). The instrument allowed clinicians to view a computer monitor displaying visual force-feedback information in real time while performing MCD. The monitor displayed a cursor which represented traction force. The visual feedback tool was implemented in the RCT on 27 June 2013. Clinicians provided a total of $231 \mathrm{MCD}$ treatments without visual feedback and 218 treatments with visual feedback. We reported the impact of this protocol change on the proficiency of clinician traction force delivery elsewhere [45].

\section{Discussion}

This paper presents the results of a pilot randomized clinical trial of MCD for patients with chronic neck pain designed to evaluate an attention-touch control for future clinical trials. MCD is a traction-based treatment for cervical and thoracic spine pain and dysfunction that is commonly delivered by DCs in the USA [79]. Potential advantages of MCD over other types of traction include its delivery in the prone position, which may enhance the localization of manual forces applied more specifically to a region of the spine compared with other treatments that apply traction via straps around the mandible and occipital region of the head. To our knowledge, no other studies have investigated MCD using a force-based approach or assessed the traction forces delivered during MCD using biomechanical measures. In addition, the technological advancement represented by real-time force feedback enhances the feasibility of consistent treatment delivery force [46].

One advantage of this study was its mixed methods approach to the development of a minimal intervention control for RCTs of traction-based therapies. Manual therapy research has been hampered by the lack of viable control procedures [32, 80-82]. As manual therapies require physical contact between the clinician and 
patient, it is difficult to introduce a believable sham or minimal intervention that is not immediately obvious to participants or that has no specific effect on the clinical outcomes under investigation [81]. Vernon and colleagues developed a sham cervical procedure that $50 \%$ of participants suspected was real SM, and that had no clinically important changes in cervical range of motion, pain or tenderness [83]. However, this sham procedure is a high-velocity spinal manipulation maneuver, quite unlike the gradual spinal mobilization of MCD, making it ill-suited as a control intervention for studies of traction procedures.

We developed a traction force-based minimal intervention that demonstrated some characteristics of a successful attention-touch control group, such as the lack of clinically important changes in the primary outcomes. Participants who received the low force treatment did identify this procedure as the minimal intervention and stated their assessment was due to the lightness of the clinician's touch. Further, many medium force participants incorrectly guessed that they had received the low force intervention. As our team measured the amount of traction force participants received at each treatment visit, it was possible to identify the mean traction forces delivered to each group. Based on these combined qualitative and traction force data, we will modify the force ranges for the minimal intervention and MCD groups in future studies. An increase in the minimal intervention traction force range from 0 to $20 \mathrm{~N}$ to 15 to $30 \mathrm{~N}$ should enhance treatment credibility (that is, the lightness of the clinician's touch) while still minimizing the treatment effect. In this pilot, the traction force range for the high force group was 51 to $100 \mathrm{~N}$. To maximize the distinction between a minimal intervention and the MCD group in future trials, we will set the MCD traction force range at 60 to $100 \mathrm{~N}$, which is consistent with the peak forces measured in the high force group, as well as participants' qualitative assessments of treatment delivery in this group. Future trials should conduct similar qualitative assessment to ascertain whether these modifications adequately enhance the credibility of the minimal intervention control.

The changes in patient-reported outcomes during this pilot were promising. Group differences were demonstrated on the neck pain VAS and NDI for the medium force and high force groups compared to the low force group. Unexpected, but intriguing changes were the group differences noted in fatigue and sleep disturbance outcomes. However, differences in the primary and secondary outcomes between the medium and high force groups were negligible. In part, this finding may be due the overlap in traction forces between groups noted midway through the trial which was resolved by the use of visual force feedback technology [46]. Future studies of MCD might opt to compare treatment groups with more distinct differences such as Snodgrass et al. reported in a pilot study utilizing a force-based posterior-toanterior mobilization procedure [84]. In that study, significant reductions in pain and spinal stiffness were reported in participants receiving the high force posterior-toanterior mobilization $(90 \mathrm{~N})$ compared to low force $(30 \mathrm{~N})$ or placebo [84]. As the greatest improvements in neck pain severity and neck-related disability in our study were reported by the high traction force group, a fully powered future RCT might compare high force traction to a minimal intervention, attention-touch control.

Of note, the improvements in PROs reported in this study were achieved in a pilot RCT that was only two weeks in duration. Future RCTs that evaluate MCD might extend the course of care to four or six weeks, as is delivered in clinical practice [34] and in recent clinical trials of traction-based therapies which demonstrated both shortterm and longer term improvements in neck pain intensity and disability following four weeks of treatment $[24,85]$.

Additional aims of this pilot study were to assess the feasibility of the participant recruitment plan and to evaluate the safety of force-based delivery of MCD. Community interest in this trial was good as evidenced by the consistent participant recruitment, enrollment and trial completion. We enrolled a middle-aged sample with the majority of participants being women, which is consistent with the patient population that reports musculoskeletal neck pain $[7,8]$. Data collection took nine months, nearly three months sooner than anticipated during study development. Few participants missed any visits, only three participants withdrew from the trial, and none were from the low force group. These results support the feasibility of our participant recruitment plan for a fully-powered randomized controlled trial. Additional recruitment methods that might enhance enrollment during a larger trial are outreach to community groups, press releases and social media announcements, particularly those targeting populations in which neck pain is a prominent concern such as middle aged women, as well as the possible addition of financial incentives $[49,86]$. We randomized participants who reported symptoms consistent with QTF classifications 2 (neck pain with radiation to proximal extremities) and 3 (neck pain with radiation to distal extremities). In future trials of MCD, researchers may consider partnering with healthcare providers who treat persons with chronic neck pain to identify potential participants with these clinical characteristics.

During our pilot, we limited our inclusion criteria to participants who rated their neck pain at baseline as between 3 and 7 on the NRS as a safety precaution. Thus, a number of potential participants $(\mathrm{n}=32)$ were excluded for pain severity levels of 8,9 or 10 on the NRS. 
Given the relatively low number of adverse events related to study interventions reported in the trial, which were equally distributed between groups, of mild or moderate severity, and followed established patterns of adverse events from spinal mobilization reported in the literature [69, 73, 74, 87], opening eligibility criteria to include persons with greater neck pain severity levels seems acceptable and would likely increase participant eligibility for a larger trial.

This pilot RCT had several limitations. As in manual therapy trials generally [88], the clinicians who delivered the intervention were not blinded to treatment group nor were the biomechanics research assistants blinded to force measurements. Both types of personnel received extensive training and followed an explicit script to prevent the inadvertent unblinding of study participants to their treatment group. In addition, study participants were excluded if they reported high levels of neck pain, cervical fusion, and several other co-morbid conditions which are common among persons with chronic neck pain [2]. No additional measures were employed to control for the angle of distraction, which theoretically varies between participants who inherently exhibit varied morphology. Characteristics affecting the angle of distraction could include thoracic cage diameter, breast tissue, abdominal tissue, and cervico-thoracic curvature.

Our study tested a single component of MCD - traction in a neutral head position - which is thought to be the most important component contributing therapeutic benefit [34]. However, practitioners who use MCD often incorporate multiple manually delivered therapies focused on the cervical and thoracic spine into their treatment regimens. In addition, the manual contact points treated in clinical practice may differ from those in this study (C5, occiput). Also, the number of treatments provided $(n=5)$ is potentially dissimilar to the use of MCD in practice settings, in that the clinician determines the frequency and duration of treatment based on the type and severity of the condition, the patient's response to treatment, and the patient's care phase (acute, subacute, chronic, rehabilitation) [34]. Our decision to standardize these delivery components of the MCD intervention increased the rigor of this pilot RCT, but may have limited the participants' clinical response to the intervention. If the efficacy of traction with a neutral head position is established, trials to assess the full complement of the MCD intervention may be warranted.

Another limitation is the cost of the equipment used to measure traction forces. Gudavalli et al. reported on the proficiency of trained DCs demonstrating $75 \%$ proficiency with no feedback and $97 \%$ with visual feedback [46]. The technology used in this study was developed for research purposes rather than clinical application. This equipment may be cost prohibitive for many clinicians to purchase for their practice settings. The development of a low cost technology to offer force feedback in practice settings is feasible and would advance the training of manual therapists.

\section{Conclusions}

This paper reports the primary findings of a pilot randomized clinical trial of MCD for adult patients with chronic neck pain designed to develop a minimal intervention to serve as an attention-touch control for future studies. Participants who received the medium $(21 \mathrm{~N}$ to $50 \mathrm{~N})$ to high ( $51 \mathrm{~N}$ to $100 \mathrm{~N})$ traction force MCD reported clinically important improvements in neck pain intensity and neck-related disability compared to participants who received low $(\leq 20 \mathrm{~N})$ traction force MCD. These improvements in clinical outcomes were noted after only four sessions of MCD delivered over a twoweek period. Adverse events were of mild or moderate severity and self-resolved, demonstrating the safety of the intervention. These data support undertaking a fully powered, randomized controlled trial of MCD using the minimal intervention as an attention-touch control.

\section{Consent to use photographs}

Written informed consent was obtained from the patient(s) for publication of this manuscript and accompanying images. A copy of the written consent is available for review by the Editor-in-Chief of this journal.

\begin{abstract}
Abbreviations
AE: Adverse event; BL1: Baseline 1 visit; BL2: Baseline 2 visit; C5: 5th Cervical vertebra; CEQ: Credibility and expectancy questionnaire; Hz: Hertz; IMMPACT: Initiative on methods, measurement, and pain assessment in clinical trials; MCD: Manual cervical distraction; N: Newton; NDI: Neck disability index; NRS: Numerical rating scale; PROMIS: Patient reported outcomes measurement information system; RCT: Randomized clinical trial; SAE: Serious adverse event; SAS: SAS Institute, Inc.; SQL: Structured query language; SSL: Secure socket layers; SV: Study visit; VAS: Visual analogue scale.
\end{abstract}

\section{Competing interests}

SAS, RDV, LC, CRL, AGP, and CMG declare they have no competing interests. MRG declared receiving travel reimbursement for presentation of Research at Cox Technique certification seminars. Haven Innovations sold the treatment table to the Palmer Center for Chiropractic Research for research purposes at a discount of $\$ 5,000$.

\section{Authors' contributions}

$M R G, R D V, S A S, C R L, A G P$, and CMG are responsible for the conceptualization of the trial. CMG is the principal investigator of the center grant award, and MRG and AGP are project co-leaders. MRG, RDV, SAS, CRL, LC, AGP, and CMG participated in data analysis plans. MRG, CRL, and LC conducted statistical data analysis, while SAS completed the qualitative analysis. MRG, SAS, RDV and CRL drafted the manuscript while LC, AGP, and CMG provided critical review. All authors read and approved the final manuscript.

\section{Acknowledgments}

This study is supported by a grant from the National Institutes of Health, National Center for Complementary and Alternative Medicine under grant number 5U19AT004663, Developmental Center for Clinical and Translational Science in Chiropractic. This project was conducted in a facility constructed with support from Research Facilities Improvement Grant\# C06 RR015433 
from the National Center for Research Resources. The content and conclusions of this manuscript are solely the responsibility of the authors and do not necessarily represent the official views or policies of the US government or any supporting entity. The authors thank the study participants and research personnel for their involvement in this clinical trial.

\section{Author details}

${ }^{1}$ Palmer Center for Chiropractic Research, Palmer College of Chiropractic, 741 Brady Street, 52803 Davenport, IA, USA. ${ }^{2}$ Musculoskeletal Biomechanics Laboratory, Edward Hines Jr. VA Hospital, Hines, IL and Department of Orthopedic Surgery \& Rehabilitation, Stritch School of Medicine, Loyola University Chicago, Chicago, IL, USA.

Received: 12 November 2014 Accepted: 20 May 2015

Published online: 05 June 2015

\section{References}

1. Blozik E, Laptinskaya D, Herrmann-Lingen C, Schaefer H, Kochen M, Himmel W, et al. Depression and anxiety as major determinants of neck pain: a cross-sectional study in general practice. BMC Musculoskelet Disord. 2009;10:13.

2. Carroll LJ, Hogg-Johnson S, Velde G, Haldeman S, Holm LW, Carragee EJ, et al. Course and prognostic factors for neck pain in the general population: results of the Bone and Joint Decade 2000-2010 Task Force on Neck Pain and Its Associated Disorders. Spine. 2008;33:575-82.

3. O'Neill KR, Wilson RJ, Burns KM, Mioton LM, Wright BT, Adogwa O, McGirt MJ, Devin CJ: Anterior cervical discectomy and fusion for adjacent-segment disease: clinical outcomes and cost-utility of surgical intervention. J Spinal Disord Tech 2013, Epub.

4. Hoy DG, Protani M, De R, Buchbinder R. The epidemiology of neck pain. Best Pract Res Clin Rheumatol. 2010;24:783-92.

5. Hogg-Johnson S, van der Velde, Carroll L, Holm LW, Cassidy JD, Guzman J, et al. The burden and determinants of neck pain in the general population: results of the Bone and Joint Decade 2000-2010 Task Force on Neck Pain and Its Associated Disorders. J Manipulative Physiol Ther. 2009;32 Suppl 2:S46-60.

6. Cagnie B, Danneels L, Van Tiggelen D, Loose V, Cambier D. Individual and work related risk factors for neck pain among office workers: a cross sectional study. Eur Spine J. 2007;16:679-86.

7. Croft PR, Lewis M, Papageorgiou AC, Thomas E, Jayson MI, Macfarlane GJ, et al. Risk factors for neck pain: a longitudinal study in the general population. Pain. 2001;93:317-25.

8. Skillgate $E$, Magnusson C, Lundberg M, Hallqvist J. The age- and sex-specific occurrence of bothersome neck pain in the general population-results from the Stockholm public health cohort. BMC Musculoskelet Disord. 2012;13:185.

9. Riddle DL, Schappert SM. Volume and characteristics of inpatient and ambulatory medical care for neck pain in the United States: data from three national surveys. Spine. 2007:32:132-40

10. Cote P, Cassidy JD, Carroll L. The treatment of neck and low back pain: who seeks care? who goes where? Med Care. 2001;39:956-67.

11. Martin BI, Deyo RA, Mirza SK, Turner JA, Comstock BA, Hollingworth W, et al. Expenditures and health status among adults with back and neck problems. JAMA. 2008:299:656-64.

12. Martin BI, Turner JA, Mirza SK, Lee MJ, Comstock BA, Deyo RA. Trends in health care expenditures, utilization, and health status among US adults with spine problems, 1997-2006. Spine. 2009;34:2077-84.

13. Simon LS. Nonsteroidal anti-inflammatory drugs and their risk: a story still in development. Arthritis Res Ther. 2013;15 Suppl 3:S1.

14. Wehling M. Non-steroidal anti-inflammatory drug use in chronic pain conditions with special emphasis on the elderly and patients with relevant comorbidities: management and mitigation of risks and adverse effects. Eur J Clin Pharmacol. 2014;70:1159-72.

15. Duarte R, Raphael J. The pros and cons of long-term opioid therapy. J Pain Palliat Care Pharmacother. 2014;28:308-10.

16. Gross AR, Hoving JL, Haines TA, Goldsmith CH, Kay T, Aker P, et al. A Cochrane review of manipulation and mobilization for mechanical neck disorders. Spine. 2004;29:1541-8.

17. Gross A, Miller J, D'Sylva J, Burnie SJ, Goldsmith CH, Graham N, et al. Manipulation or mobilisation for neck pain: a Cochrane review. Man Ther. 2010;15:315-33.

18. Bronfort G, Haas M, Evans R, Leininger B, Triano J. Effectiveness of manual therapies: the UK evidence report. Chiropr Osteopat. 2010;18:3.
19. Miller J, Gross A, D'Sylva J, Burnie SJ, Goldsmith CH, Graham N, et al. Manual therapy and exercise for neck pain: a systematic review. Man Ther. 2010;15:334-54.

20. Sutton DA, Cote P, Wong JJ, Varatharajan S, Randhawa KA, Yu H, Southerst D, Shearer HM, van der Velde GM, Nordin MC, Carroll LJ, Mior SA, Taylor-Vaisey AL, Stupar M. Is multimodal care effective for the management of patients with whiplash-associated disorders or neck pain and associated disorders? A systematic review by the Ontario Protocol for Traffic Injury Management (OPTIMa) Collaboration. Spine J. 2014. doi:10.1016/j.spinee.2014.06.019. Epub.

21. Goldie I, Landquist A. Evaluation of the effects of different forms of physiotherapy in cervical pain. Scand J Rehabil Med. 1970;2:117-21.

22. Zylbergold RS, Piper MC. Cervical spine disorders. A comparison of three types of traction. Spine. 1985;10:867-71.

23. Chiu TT, Ng JK, Walther-Zhang B, Lin RJ, Ortelli L, Chua SK. A randomized controlled trial on the efficacy of intermittent cervical traction for patients with chronic neck pain. Clin Rehabil. 2011;25:814-22.

24. Fritz JM, Thackeray A, Brennan GP, Childs JD. Exercise only, exercise with mechanical traction, or exercise with over-door traction for patients with cervical radiculopathy, with or without consideration of status on a previously described subgrouping rule: a randomized clinical trial. J Orthop Sports Phys Ther. 2014:44:45-57.

25. Young IA, Michener LA, Cleland JA, Aguilera AJ, Snyder AR. Manual therapy, exercise, and traction for patients with cervical radiculopathy: a randomized clinical trial. Phys Ther. 2009;89:632-42.

26. Graham N, Gross A, Goldsmith CH, Klaber MJ, Haines T, Burnie SJ, et al. Mechanical traction for neck pain with or without radiculopathy. Cochrane Database Syst Rev. 2008;3, CD006408.

27. Patel KC, Gross A, Graham N, Goldsmith CH, Ezzo J, Morien A, et al. Massage for mechanical neck disorders. Cochrane Database Syst Rev. 2012;9, CD004871. doi:10.1002/14651858.CD004871.

28. Briem K, Huijbregts $P$, Thorsteinsdottir M. Immediate effects of inhibitive distraction on active range of cervical flexion in patients with neck pain: a pilot study. J Man Manip Ther. 2007;15:82-92.

29. Hanten WP, Olson SL, Butts NL, Nowicki AL. Effectiveness of a home program of ischemic pressure followed by sustained stretch for treatment of myofascial trigger points. Phys Ther. 2000;80:997-1003.

30. Zaproudina N, Hanninen OO, Airaksinen O. Effectiveness of traditional bone setting in chronic neck pain: randomized clinical trial. J Manipulative Physiol Ther. 2007;30:432-7.

31. Sherman KJ, Cook AJ, Wellman RD, Hawkes RJ, Kahn JR, Deyo RA, et al. Five-week outcomes from a dosing trial of therapeutic massage for chronic neck pain. Ann Fam Med. 2014;12:112-20.

32. Haines T, Gross AR, Burnie S, Goldsmith CH, Perry L, Graham N. A Cochrane review of patient education for neck pain. Spine J. 2009;9:859-71.

33. Evans DW, Lucas N. What is 'manipulation'? A reappraisal Man Ther. 2010;15:286-91.

34. Cox JM. Neck, Shoulder, arm pain: mechanism, diagnosis, treatment. 3rd ed. Fort Wayne: Cox ${ }^{\circledast}$ Technic Resource Center Inc; 2004.

35. Cox JM, Aspegren DD. Degenerative spondylolisthesis of $C 7$ and $L 4$ in same patient. J Manipulative Physiol Ther. 1988;11:195-205.

36. Gudavalli S, Kruse RA. Foraminal stenosis with radiculopathy from a cervical disc herniation in a 33-year-old man treated with flexion distraction decompression manipulation. J Manipulative Physiol Ther. 2008;31:376-80.

37. Kruse RA, Cambron JA. Large C4/5 spondylotic disc bulge resulting in spinal stenosis and myelomalacia in a Klippel-Feil patient. J Altern Complement Med. 2012;18:96-9.

38. Kruse RA, Imbarlina F, De Bono VF. Treatment of cervical radiculopathy with flexion distraction. J Manipulative Physiol Ther. 2001;24:206-9.

39. Schliesser JS, Kruse R, Fallon LF. Cervical radiculopathy treated with chiropractic flexion distraction manipulation: a retrospective study in a private practice setting. J Manipulative Physiol Ther. 2003;26, E19.

40. Gudavalli MR, Potluri T, Carandang G, Havey RM, Voronov LI, Cox JM, et al. Intradiscal pressure changes during manual cervical distraction: a cadaveric study. Evid Based Complement Alternat Med. 2013;2013:954134.

41. Gudavalli MR, Henderson CN, Vining RD, Carber L, Patwardhan AG, Goertz C. Neck pain participants' perceptions of traction forces during chiropractic manual cervical distraction. In: Proceedings of the ASME 2012 International Mechanical Engineering Congress and Exposition. Biomedical and Biotechnology. 2012;2:53-4. doi:10.1115/IMECE2012-85971.

42. Gudavalli MR, Yadav V, Vining R, Seidman M, Salsbury S, Morgenthal $P$, et al. Development of force-feedback technology for training clinicians to deliver manual cervical distraction. In: Proceedings of the 2013 International 
Mechanical Engineering Congress and Exposition November, 2013. San Diego, CA, USA: Volume 3A: Biomedical and Biotechnology Engineering; 2013. doi:10.1115/IMECE2013-64509.

43. Gudavalli MR, Vining RD, Salsbury SA, Goertz CM. Training and certification of doctors of chiropractic in delivering manual cervical traction forces: results of a longitudinal observational study. J Chiropr Educ. 2014;28:130-8.

44. Thabane L, Ma J, Chu R, Cheng J, Ismaila A, Rios L, et al. A tutorial on pilot studies: the what, why and how. BMC Med Res Methodol. 2010;10:1.

45. Gudavalli MR, Vining RD, Salsbury SA, Corber L, Long CR, Patwardhan AG, et al. Doctors of chiropractic proficiency in delivering prescribed traction forces during manual cervical distraction of neck pain patients. J Altern Complement Med. 2014;20:A48-9.

46. Gudavalli MR, Vining RD, Salsbury SA, Corber LG, Long CR, Patwardhan AG, et al. Clinician proficiency in delivering manual treatment for neck pain within specified force ranges. Spine J. 2015;15:570-6.

47. Friedman L. Commentary: why we should report results from clinical trial pilot studies. Trials. 2013;14:14.

48. Twist E, Lawrence DJ, Salsbury SA, Hawk C. Do informed consent documents for chiropractic clinical research studies meet readability level recommendations and contain required elements: a descriptive study. Chiropr Man Ther. 2014;22:40.

49. Hondras MA, Long CR, Haan AG, Spencer LB, Meeker WC. Recruitment and enrollment for the simultaneous conduct of 2 randomized controlled trials for patients with subacute and chronic low back pain at a CAM research center. J Altern Complement Med. 2008;14:983-92.

50. Vining R, Salsbury S, Pohlman K. Eligibility determination for clinical trials: development of a case review process at a chiropractic research center. Trials. 2014;15:406

51. Khorsan R, Coulter ID, Hawk C, Choate CG. Measures in chiropractic research: choosing patient-based outcome assessments. J Manipulative Physiol Ther. 2008;31:355-75.

52. Schulz KF, Altman DG, Moher D. CONSORT 2010 Statement: updated guidelines for reporting parallel group randomised trials. BMC Med. 2010;8:18.

53. Beck AT, Steer RA, Carbin MG. Psychometric properties of the Beck depression inventory: twenty-five years of evaluation. Clin Psychol Rev. 1988:8:77-100.

54. Bolton JE, Wilkinson RC. Responsiveness of pain scales: a comparison of three pain intensity measures in chiropractic patients. J Manipulative Physiol Ther. 1998:21:1-7.

55. Hawker GA, Mian S, Kendzerska T, French M. Measures of adult pain: Visual Analog Scale for Pain (VAS Pain), Numeric Rating Scale for Pain (NRS Pain), McGill Pain Questionnaire (MPQ), Short-Form McGill Pain Questionnaire (SF-MPQ), Chronic Pain Grade Scale (CPGS), Short Form-36 Bodily Pain Scale (SF-36 BPS), and Measure of Intermittent and Constant Osteoarthritis Pain (ICOAP). Arthritis Rheumatism. 2011;63 Suppl 11:S240-52

56. Vernon $\mathrm{H}$, Mior S. The neck disability index: a study of reliability and validity. J Manipulative Physiol Ther. 1991;14:409-15.

57. Fairbank JC, Pynsent PB. The Oswestry disability index. Spine. 2000;25:2940-52.

58. Dworkin RH, Turk DC, Farrar JT, Haythornthwaite JA, Jensen MP, Katz NP, et al. Core outcome measures for chronic pain clinical trials: IMMPACT recommendations. Pain. 2005;113:9-19.

59. Cella D, Riley W, Stone A, Rothrock N, Reeve B, Yount S, et al. Initial adult health item banks and first wave testing of the Patient-Reported Outcomes Measurement Information System (PROMIS) Network: 2005-2008. J Clin Epidemiol. 2010;63:1179.

60. Smeets RJ, Beelen S, Goossens ME, Schouten EG, Knottnerus JA, Vlaeyen JW. Treatment expectancy and credibility are associated with the outcome of both physical and cognitive-behavioral treatment in chronic low back pain. Clin J Pain. 2008:24:305-15.

61. Devilly GJ, Borkovec TD. Psychometric properties of the credibility/expectancy questionnaire. J Behav Ther Exp Psychiatry. 2000;31:73-86.

62. Cambron JA, Jennifer Dexheimer LM, Cramer GD. Patients' perception of care: comparing placebo and manual therapy within a pilot study. Topics in Integrative Health Care. 2003;2:2.

63. Hsieh HF, Shannon SE. Three approaches to qualitative content analysis. Qual Health Res. 2005;15:1277-88.

64. Hsieh HF, Shannon SE. Three approaches to qualitative content analysis. Qual Health Res. 2005;15:1277-88.

65. Goertz CM, Salsbury SA, Vining RD, Long CR, Andresen AA, Jones ME, et al. Collaborative Care for Older Adults with low back pain by family medicine physicians and doctors of chiropractic (COCOA): study protocol for a randomized controlled trial. Trials. 2013;14:18.
66. Wilder DG, Vining RD, Pohlman KA, Meeker WC, Xia T, DeVocht JW, et al. Effect of spinal manipulation on sensorimotor functions in back pain patients: study protocol for a randomised controlled trial. Trials. 2011;12:161.

67. Carnes D, Mars TS, Mullinger B, Froud R, Underwood M. Adverse events and manual therapy: a systematic review. Man Ther. 2010;15:355-63.

68. Carnes D, Mullinger B, Underwood M. Defining adverse events in manual therapies: a modified Delphi consensus study. Man Ther. 2010;15:2-6.

69. Carlesso LC, Gross AR, Santaguida PL, Burnie S, Voth S, Sadi J. Adverse events associated with the use of cervical manipulation and mobilization for the treatment of neck pain in adults: a systematic review. Man Ther. 2010;15:434-44

70. Carlesso LC, MacDermid JC, Santaguida LP. Standardization of adverse event terminology and reporting in orthopaedic physical therapy: application to the cervical spine. J Orthop Sports Phys Ther. 2010:40:455-63.

71. Ioannidis JP, Evans SJ, Gotzsche PC, O'Neill RT, Altman DG, Schulz K, et al. Better reporting of harms in randomized trials: an extension of the CONSORT statement. Ann Intern Med. 2004:141:781-8.

72. National Institutes of Health. Guidance on reporting adverse events to institutiona review boards for $\mathrm{NIH}$-supported multicenter clinical trials. Bethesda: National Institutes of Health; 1999. p. 6.

73. Hurwitz EL, Morgenstern H, Vassilaki M, Chiang LM. Adverse reactions to chiropractic treatment and their effects on satisfaction and clinical outcomes among patients enrolled in the UCLA Neck Pain Study. J Manipulative Physio Ther. 2004;27:16-25.

74. Eriksen K, Rochester RP, Hurwitz EL. Symptomatic reactions, clinical outcomes and patient satisfaction associated with upper cervical chiropractic care: a prospective, multicenter, cohort study. BMC Musculoskelet Disord. 2011:12:219.

75. Spritzer WO, Leblanc FE, Dupuis M. Scientific approach to the assessment and management of activity-related spinal disorders. A monograph for clinicians. Report of the Quebec Task Force on Spinal Disorders. Spine. 1987;12 Suppl 7:S1-59

76. Guzman J, Hurwitz EL, Carroll L, Haldeman S, Cote P, Carragee EJ, et al. A new conceptual model of neck pain: linking onset, course, and care: the Bone and Joint Decade 2000-2010 Task Force on Neck Pain and its Associated Disorders. Spine. 2008;33 Suppl 4:S14-23.

77. Vernon H. The Neck Disability Index: state-of-the-art, 1991-2008. J Manipulative Physiol Ther. 2008:31:491-502.

78. Marshall B, Cardon P, Poddar A, Fontenot R. Does sample size matter in qualitative research: a review of qualitative interviews in IS research. J Computer Information Syst. 2013:54:11-22

79. Christensen MG, Mark G, Kollash MW, Hyland JK. Practice Analysis of Chiropractic 2010: a project report, survey analysis, and summary of chiropractic practice in the United States. National Board of Chiropractic Examiners: Greeley, CO; 2010.

80. Moyer CA, Rounds J, Hannum JW. A meta-analysis of massage therapy research. Psychol Bull. 2004;130:3-18.

81. Nahin RL, Straus SE. Research into complementary and alternative medicine: problems and potential. BMJ. 2001;322:161-4.

82. Gross A, Miller J, D'Sylva J, Burnie SJ, Goldsmith CH, Graham N, et al. Manipulation or mobilisation for neck pain. Cochrane Database Syst Rev. 2010;1, CD004249.

83. Vernon HT, Triano JJ, Ross JK, Tran SK, Soave DM, Dinulos MD. Validation of a novel sham cervical manipulation procedure. Spine J. 2012;12:1021-8.

84. Snodgrass SJ, Rivett DA, Sterling M, Vicenzino B. Dose optimization for spinal treatment effectiveness: a randomized controlled trial investigating the effects of high and low mobilization forces in patients with neck pain. J Orthop Sports Phys Ther. 2014;44:141-52.

85. Moustafa IM, Diab AA. Multimodal treatment program comparing 2 different traction approaches for patients with discogenic cervical radiculopathy: a randomized controlled trial. J Chiropr Med. 2014;13:157-67.

86. Gupta A, Calfas KJ, Marshall SJ, Robinson TN, Rock CL, Huang JS, et al. Clinical trial management of participant recruitment, enrollment, engagement, and retention in the SMART study using a Marketing and Information Technology (MARKIT) model. Contemp Clin Trials. 2015;42:185-95.

87. Hurwitz EL, Morgenstern H, Vassilaki M, Chiang LM. Frequency and clinical predictors of adverse reactions to chiropractic care in the UCLA neck pain study. Spine (Phila Pa 1976). 2005;30:1477-84.

88. Licciardone JC, Minotti DE, Gatchel RJ, Kearns CM, Singh KP. Osteopathic manual treatment and ultrasound therapy for chronic low back pain: a randomized controlled trial. Ann Fam Med. 2013;11:122-9. 